\title{
APPLICATION OF VIDEO IMAGES FOR MONITORING COASTAL ZONE IN HASAKI BEACH, JAPAN
}

\author{
Muhammad Zikra', Noriaki Hashimoto², Masaru Yamashiro², Masaki Yokota² and \\ Kojiro Suzuki ${ }^{3}$
}

\begin{abstract}
The video image analysis methods are used for monitoring the phenomena at the coastal zone. A digital video camera which is mounted in the HORS research building is used to estimate directional wave spectra and bathymetry in shallow water areas. The Bayesian Directional Method is used to estimate directional wave spectra using the group of pixels brightness and non-linear bathymetry inversion is used to estimate shallow water bathymetry using time series of pixel brightness intensity at cross-shore and longshore array. The results are compared with a numerical model and field measurements. The results indicate that video image data could be used to estimate bathymetry and directional wave spectra in shallow water areas.
\end{abstract}

Keywords: bathymetry; directional wave spectra; wavenumber; video images

\section{INTRODUCTION}

Since the first development of the video image method in 1980 by Coastal Imaging Lab, Oregon State University, USA, the capabilities of video remote sensing techniques have been developed and applied into very useful tools for monitoring coastal changes in the nearshore environment area (Aarninkhof and Holman 1999). These capabilities allow for studying sandbar morphology (Lippman and Holman 1997), foreshore beach slope (Plant and Holman 1997), wave run-up (Holland and Holman 1989; Holland et al. 1997), to study wave phase or wave number to estimate bathymetry (Stockdon and Holman 2000; Plant et al. 2007), for estimation of wave directional spectra (Holman and Chickadel 2004), for studying coastal management (Davidson et al. 2007) and other applications.

The basic idea of the remote sensing from a video camera is to take a snapshot image of the instantaneous wave pattern in a coastal area with the assumption that the image brightness of each pixel in the snapshot images can be considered proportional to the intensities of the light reflected from the water surface. Since these successive snapshot images data are collected at a specific period, temporal changes of the wave field can be studied (Chou et al. 2004).

Although the video image methods have been applied at some coastal areas around the world (e.g. USA, Netherlands, Australia and etc), the methods have not been used for practical purposes yet since the applicability and accuracy of the methods are still limited. Therefore, it is importance to generate more research with other field measurement data so the methods can be applied for practical application in the near future.

The objectives of this paper are to examine the applicability of video image analysis methods through the following aims. The first aim is to develop a method for estimating directional wave spectra from video image data based on the Bayesian directional wave spectrum estimation method (BDM), which was originally developed by Hashimoto et al. (1987) for in-situ measurement using wave gauges. A major reason for the use of the BDM method is that this method is not only accurate but also robust in estimating directional wave spectra since it satisfies the two requirements, i.e., the minimization of errors and the smoothness of the energy distribution with respect to direction. Meanwhile, the second aim is to examine the wavenumber estimation method based on the crossspectral correlation for estimating shallow water bathymetry proposed by Plant et al. (2007). As reported by Plant et al. (2007), this method provides robust wavenumber estimates and error predictions. Also, this method is tolerant to noise and other forms of sampling deficiency.

The form of this paper is as follows. A brief review of the mathematical backgrounds used to describe directional wave spectra and bathymetry inversion is given in the next section. Next, we describe the details of study site and the information on wave data. Digital image processing and data analysis are then given in the following section. Finally, we analyze our application results and then summarize some final conclusions of this study.

\footnotetext{
${ }^{1}$ Ocean Engineering Department, Institut Teknologi Sepuluh Nopember (ITS), Gedung WA, Kampus ITS, Sukolilo, Surabaya, 60111, Indonesia

${ }^{2}$ Urban and Environmental Engineering Department, Kyushu University, 744 Motooka, Nishi-ku, Fukuoka city, 819 0395, Japan

${ }^{3}$ Marine Environmental Information Group, PARI, 3-1-1 Nagase, Yokosuka 239, Japan
} 


\section{BASIC THEORY}

\section{Directional wave spectra}

Directional wave spectra analysis method used in this study is the Bayesian Directional Method. In 1987, Hashimoto developed the Bayesian Directional Method (BDM) to estimate the directional wave spectrum for in-situ measurement using wave gauges. Estimation of the directional wave spectrum can be considered as a regression analysis to find the most suitable model from limited data. Details on the formulation of the BDM can be found in Hashimoto et al. (1987). In the BDM, the directional spreading function is expressed as a piecewise constant function over each segment of the directional range from 0 to $2 \pi$.

Since $G\left(\theta_{k}, f\right)>0$, then

$$
\ln G\left(\theta_{k}, f\right)=x_{k}(f), \quad(k=1, \ldots, K)
$$

it can be approximated as the following discretely constant value function

$$
G(\theta, f) \approx \sum_{k=1}^{K} \exp \left\{x_{k}(f)\right\} I_{k}(\theta)
$$

where

$$
I_{k}(\theta)=\left\{\begin{array}{l}
1:(k-1) \Delta \theta \leq \theta<k \Delta \theta \\
0: \quad \text { otherwise }
\end{array}\right.
$$

If unknown parameters $\left\{x_{k}\right\}$ are determined, then $G(\theta \mid f)$ is also determined. However, it becomes an improper inverse problem, because the number of unknown parameters $K$ is generally greater than the number of equations obtained from the observation. Therefore, it is assumed that $G(\theta \mid f)$ is a smooth continuous function with respect to the directional angle and the series $\left\{x_{k}\right\}$ can be locally approximated as a linear function with respect to $k$. That is, the following equation is considered.

$$
\sum_{k=1}^{K}\left(x_{k}-2 x_{k-1}+x_{k-2}\right)^{2} \approx 0 \quad ; \quad\left(x_{0}=x_{K}, x_{-1}=x_{K-1}\right)
$$

The smaller the value of Eq. (4), the smoother the function $G(\theta \mid f)$ becomes. Therefore, desirable estimate of $G(\theta \mid f)$ should maximize the likelihood with respect to $\left\{x_{k}\right\}$ within the range where equation (4) does not become too large. These criteria can be formulated as, using an appropriate parameter $u^{2}$, to find $\left\{x_{k}\right\}$ that minimizes

$$
\sum_{j=1}^{J}\left\{\Phi_{j}-\sum_{k=1}^{K} \alpha_{j, k} \exp \left(x_{k}\right)\right\}^{2}+u^{2}\left\{\sum_{k=1}^{K}\left(x_{k}-2 x_{k-1}+x_{k-2}\right)^{2}\right\}
$$

The value of $u^{2}$ and the estimate of $\sigma^{2}$ can be obtained by minimizing the Akaike's Bayesian Information Criterion (ABIC) (Akaike 1980) expressed as follows:

$$
\mathrm{ABIC}=-2 \ln \int L\left(x, \sigma^{2}\right) p\left(x \mid u^{2}, \sigma^{2}\right) d x
$$

Therefore, iterations with various $u^{2}$ are carried out to obtain the parameter $u^{2}$ that minimized the ABIC. Also, an optimum solution of $\left\{x_{k}\right\}$ is obtained and then substituted into Eq. (2) to yield an optimum estimation of the directional function.

\section{Non-linear bathymetry inversion model}

We applied non-linear inversion model related to the cross-spectral correlation as proposed by Plant et al (2007) to estimate wavenumbers. Once the wavenumbers have been estimated, then it can be used to estimate bathymetry through non-linear inversion of the dispersion equation. The estimation of wavenumber, $k$ is determined by using non-linear inversion model related to the cross-spectral 
correlation as proposed by Plant et al. (2007). The model for cross-spectral correlation equation is described by:

$$
C_{i, j, f}^{M O D E L}=\exp \left\{2 \pi \Delta x \sqrt{-1} \sum_{m=1}^{M} D_{i, j, m} k_{m, f} \cos \left(\alpha_{m, f}\right)\right\}
$$

where $f$ is wave frequency, $\Delta x$ is spacing between pixels, $D$ is design matrix defined on both sample domain $\left(x_{i}, x_{j}\right), \alpha$ (wave direction) and $k$ (wavenumber) as unknown model parameters.

The sample design matrix, $D$ is designed as basis function:

$$
D_{i, j, m}=\sum_{i^{\prime}=i}^{j} a_{i, m}
$$

where $a_{i, m}$ is smoothing weight of Hanning filter

$$
\begin{gathered}
a\left(r_{i, m}\right)=\left\{1-\cos \left(0.5 \pi\left[1+r_{i, m}\right]\right)\right\}^{2} \\
r_{i, m}=\left|x_{i}-x_{m}\right| L_{x}^{-1}
\end{gathered}
$$

where $L_{x}$ is smoothing lengths scale. Using observation data from timestack images, cross-spectral observation data can be acquired by applying discrete Fourier Transform to the observation to compute the cross-spectral between two pixels. (Bendat and Piersol 1986).

$$
C_{i, j, f}^{O B S}=\left\langle\tilde{I}\left(x_{i}, f\right) \tilde{I} *\left(x_{j}, f\right)\right\rangle
$$

where the tildes indicate the Fourier Transform, the asterisk indicates complex conjugate, angle brackets indicate ensemble or band averaging.

When the wavenumber is nonlinearly related to the cross-spectral correlation, a non-linear inversion method Levenberg-Marquardt (LM) (Press et al. 1992) is used to minimize the weighted squared difference between successive estimated of the model and the observations:

$$
\Delta C_{i, j, f}^{\tau}=\left\{\gamma_{i, j, f} C_{i, j, f}^{M O D E L(\tau)}-C_{i, j, f}^{O B S}\right\}
$$

where, at each iteration $\tau$, the model-observation mismatch is weighted by the observed coherence, $\gamma_{i, j, f}$. An iterative procedure starts with an initial value $k_{f, m}^{o}$ and new estimate of wavenumber model are obtained using:

$$
k_{f, m}^{\tau+1}=k_{f, m}^{\tau}+\Delta k_{f, m}^{\tau}
$$

where the variation $\Delta k_{f, m}^{\tau}$ calculated from

$$
\Delta k_{f, m}^{\tau}=\left(\left[R^{\tau}\right]^{T} R^{\tau}+\lambda^{\tau} I\right)^{-1}\left[R^{\tau}\right]^{T} \Delta C_{i, j, f}^{\tau}
$$

where $\lambda^{\tau}$ is the damping parameter, $I$ is the identity matrix and $R$ is sensitivity matrix for the crossspectral correlation as describe:

$$
R^{\tau}=\gamma_{i, j, f} \sqrt{-1} D_{i, j, m} C_{i, j, f}^{M O D E L(\tau)} \Delta x
$$

The iterative procedure of sequentially calculating $\Delta k_{f, m}^{\tau}$ and $k_{f, m}^{\tau+1}$ from Eq. (3.15) and (3.16) is continued until the convergence criterion $\left|\Delta k_{f, m}^{\tau}\right|<\mathcal{E}$ is satisfied, where $\varepsilon$ in this model is $10^{-6}$.

The bathymetry inversion model based on timestack method computes water depth by relating wavenumber parameters $k$ using the dispersion equation. Water depth $h$ is related to local wavenumber 
$k$ and frequency $f$ through the dispersion relationship in the linear wave theory (Dean and Dalrymple 1991)

$$
(2 \pi f)^{2}=g k \tanh (k h)
$$

where $g$ is gravitational acceleration and $h$ is local water depth.

Given a value for $f$ (sample wave frequencies) and an initial depth, $h$, this equation can be solved iteratively for wavenumber. The Levenberg-Marquardt non-linear inverse method (Press et al. 1992) was used again to minimize error between the wavenumber predicted by the dispersion relationship and the wavenumber estimated by video images. Details on the formulation of this method can be found in Plant et al. (2007).

\section{DATA}

This research study investigated video camera observations from Hasaki Beach in Japan. The data for this research was provided by the Port and Airport Research Institute (PARI), Japan. Since 1986, many coastal studies have been conducted in this location especially around the pier, which is known as Hasaki Oceanographical Research Station (HORS). The beach itself is located $120 \mathrm{~km}$ east of Tokyo, facing the North Pacific Ocean as shown in Fig. 1.

In Hasaki Beach, bathymetric survey measurements were periodically conducted once or twice a year near HORS pier within an area about $600 \mathrm{~m}$ wide in the alongshore direction and $700 \mathrm{~m}$ long in the cross-shore direction. The map of a bathymetry survey conducted in August 2006 is shown in Fig. 2. The HORS pier is located at $x=0 \mathrm{~m}$ where in-situ wave pressure gauges were installed. The HORS pier has deck dimensions of about $427 \mathrm{~m}$ long with $3.3 \mathrm{~m}$ width.

The actual surface fluctuations at the Hasaki site were recorded using several ultrasonic wave gauges. The ultrasonic wave gauges were installed on the pier with position $x=370 \mathrm{~m}, x=230 \mathrm{~m}$, and $x=145 \mathrm{~m}$ from the shoreline. Water surface fluctuations were recorded for 60 -minute segments, each of which contained approximately 7200 data points at a sampling rate of $2 \mathrm{~Hz}$.

Meanwhile, image data was collected by using a single camera installed at HORS pier in 2006. This digital video camera had a resolution of $640 \times 420$ pixels and was used to acquire snapshot images. The video camera was mounted $10 \mathrm{~m}$ above the ground level. The video image data was recorded for 15-minute durations at every 1-hour interval with a frequency sampling interval of $1 \mathrm{~Hz}$.

In 2006, the yearly average significant wave height $\mathrm{H}$ was found to be about $1.06 \mathrm{~m}$ with a corresponding wave period $T$ of $8.4 \mathrm{~s}$, based on the Nationwide Ocean Wave Information network for Port and HArborS (NOWPHAS) station at Kashima Port. During normal conditions, waves approach the coast most often from the east and southeast directions. However, the largest storm waves, with $H$ greater than 2-5 m, are incident from the northwest direction. The average of the tidal range is about $1.60 \mathrm{~m}$.
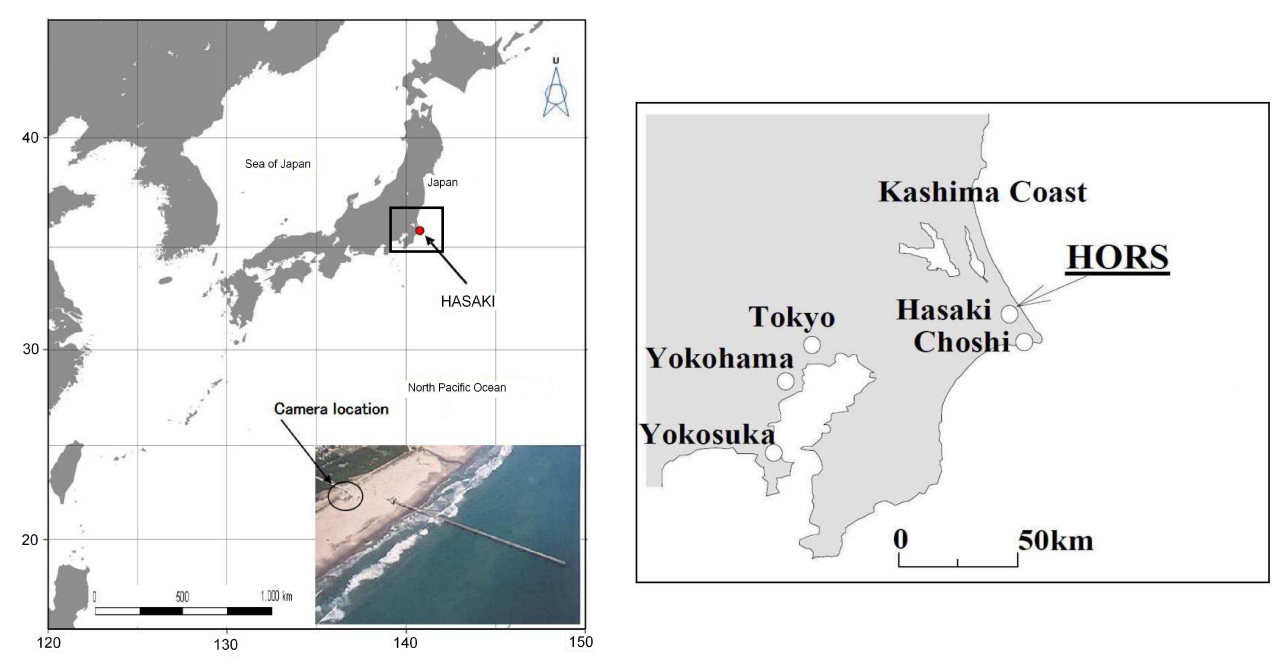

Figure 1. Map of the Hasaki site 

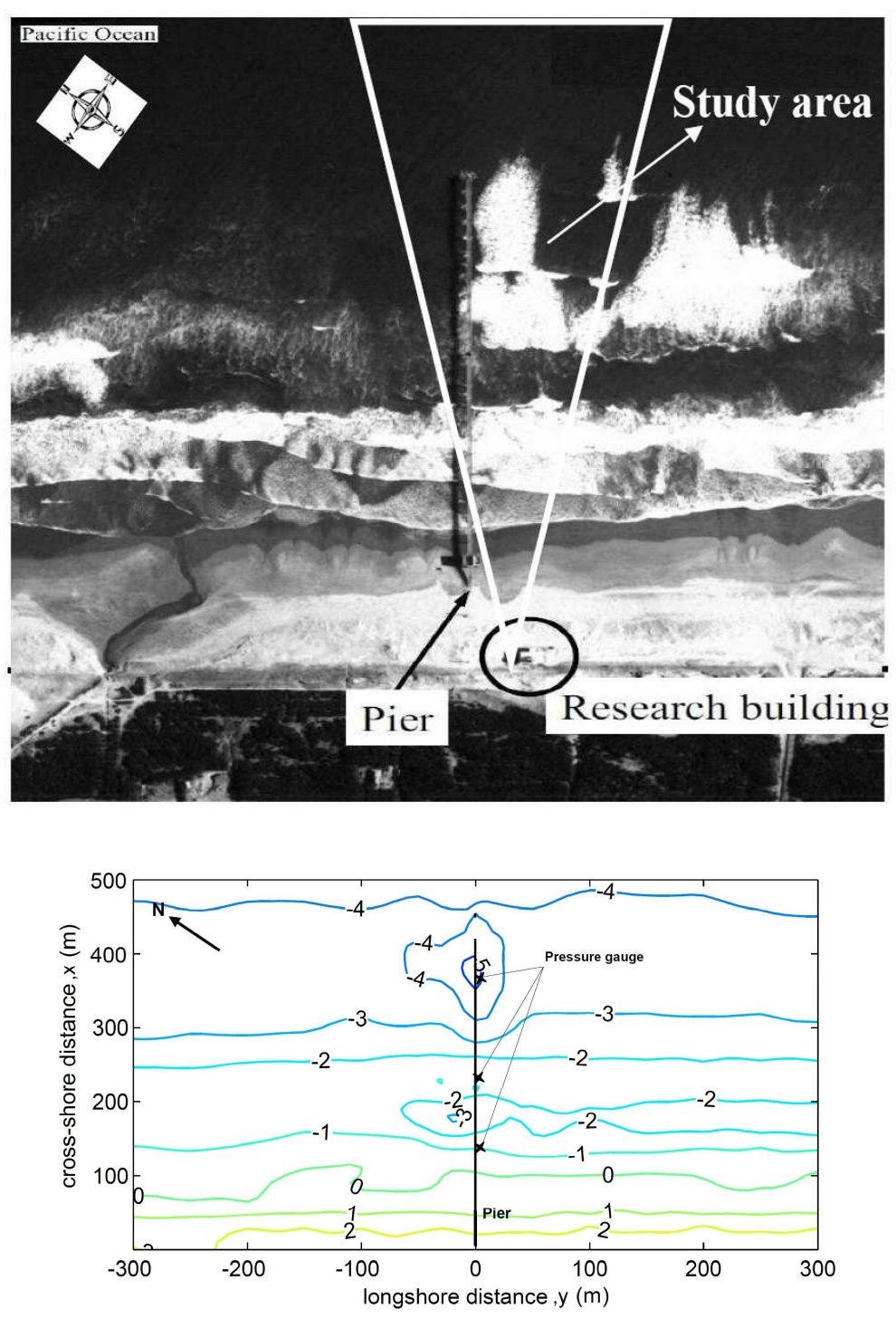

Figure 2. An aerial photo of the HORS site shows the camera view angle indicated by the triangle area (top). Bathymetry survey data measured at the HORS in August 2006 (bottom).

\section{IMAGE ANALYSIS}

First, rectification of the image must be carried out in order to extract quantitative data from a sequence of snapshot images. Figure 3 shows example of snapshot image time series. Rectification involves photogrammetric transformations, which convert image coordinates $(u, v)$ into real world coordinates $(x, y, z)$. This transformation was based on the standard photographic method as proposed and described by Holland et al (1997).

From the rectified images sequence as shown in Fig. 4, time series of pixel brightness intensity at specific points where the wave gauge is located can then be formed. Fig. 5 shows the examples of time series at $x=230 \mathrm{~m}$ (outside breaking area) from the wave gauge and pixel brightness on the video images sequence, which correspond to the nearest location of the wave gauge.

The time series of pixel brightness on video images may have introduced a source of error corresponding to the pixel resolution resulting from the rectification procedure. This is because the actual area occupied by a pixel in the image will increase with increasing distance from the camera. Figure 6 shows scale distortion resulting from a rectified image. Accuracy of rectification analysis depends on camera distance and position. Therefore, the coastal video system must be mounted as high as possible in such a way that view angles are perpendicular to the water surface. 


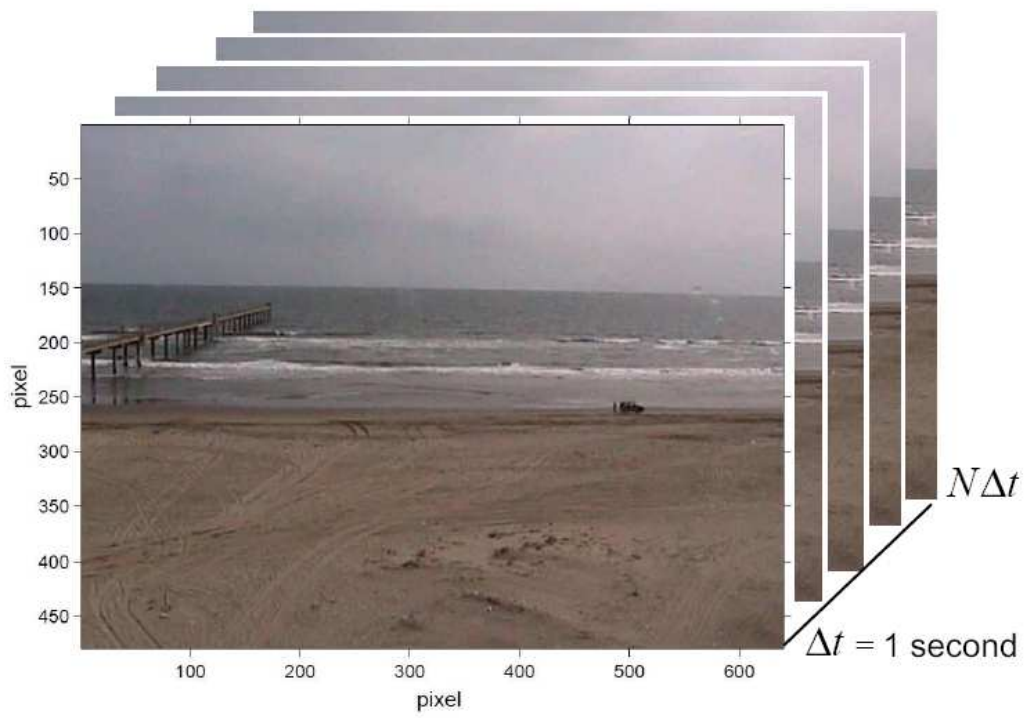

Figure 3. Snapshot image time series

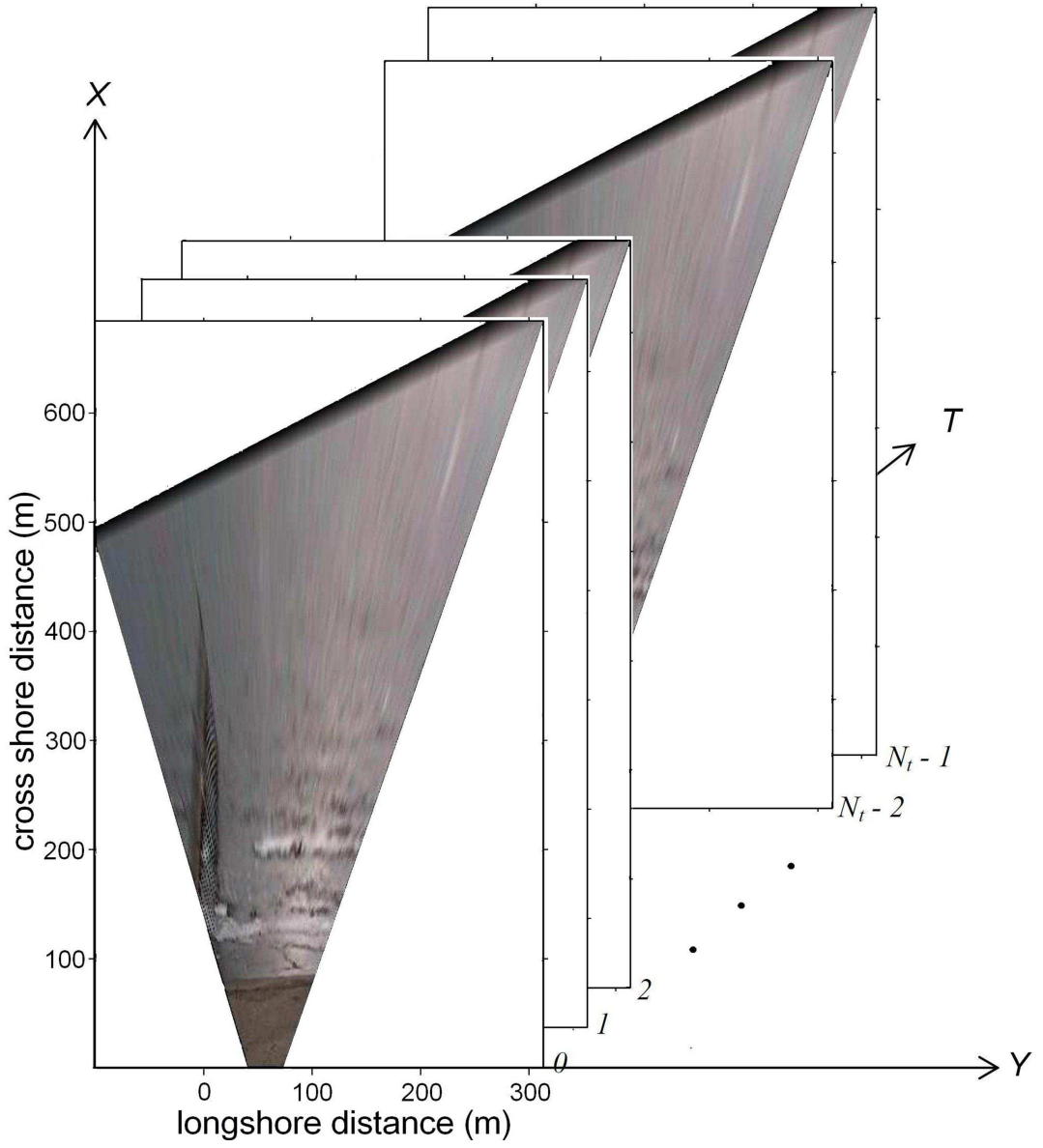

Figure 4. Rectified image time series 


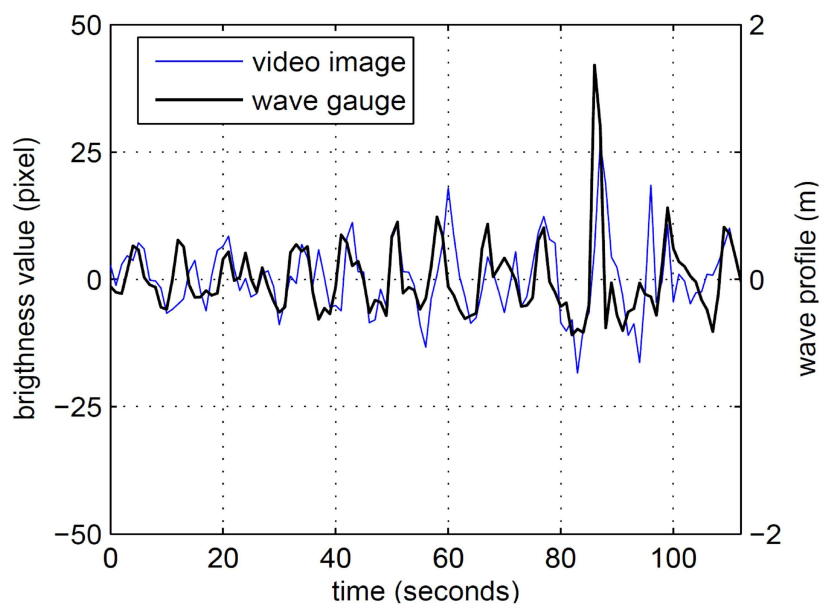

Figure 5. Comparison of time series of wave profiles from a wave gauge with sampling frequency of $0.5 \mathrm{~Hz}$ and video images with sampling frequency of $1.0 \mathrm{~Hz}$

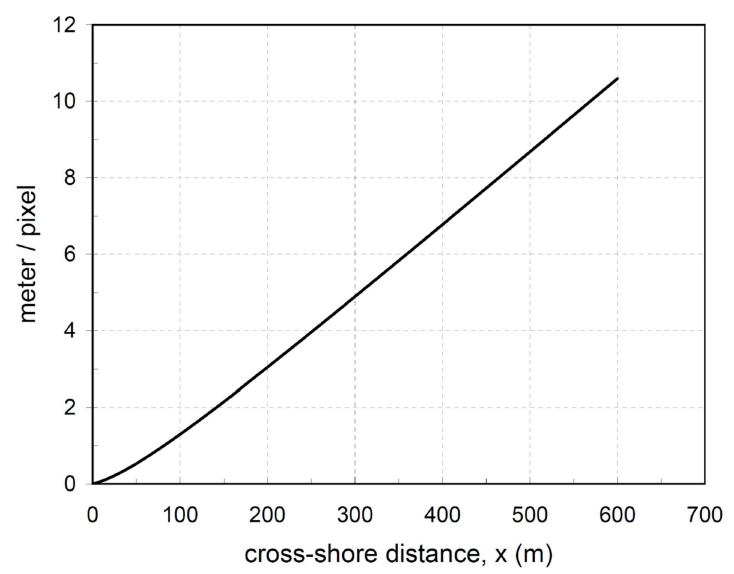

Figure 6. Scale distortion resulting from rectified images

\section{RESULTS}

\section{Directional Wave Spectra}

Video images recorded on 18 August 2006 were used in this study. On this day, the observed significant wave height based on the NOWPHAS database was below $1.0 \mathrm{~m}$, and the waves approached from the NE direction. Time series of pixel brightness on video images data was used to determine wave direction through directional wave spectra. In this study, the pixels could be considered equivalent to fixed instruments, such as a wave probe sensor, through use of the rectification process.

First, comparison of wave spectra between time series of pixel brightness on video images and insitu measurement located at $x=230 \mathrm{~m}$ is shown in Fig. 7. In order to make them comparable, we have used their respective peak spectral densities for normalization, since the energies of the wave spectra of the pixel values and wave profile measurements were measured in different scales. It can be seen that the normalized spectra are similar in shape. The figure shows a narrow band spectrum with major sharp peak at frequency $0.117 \mathrm{~Hz}$, which corresponds to a peak period of 8.5 seconds. This peak frequency corresponds with the peak period from the offshore measurement $(T=8.2$ seconds). Meanwhile, multiple weaker peaks appear at higher frequencies from $0.2 \mathrm{~Hz}$ to $0.4 \mathrm{~Hz}$. These multiple weaker peaks are present due to a nonlinear wave component, which is a typical characteristic of shallow water spectra. Below $0.05 \mathrm{~Hz}$ and above $0.4 \mathrm{~Hz}$, the white noise decreases due to band-pass filter with a high pass filter and a low pass filter. 


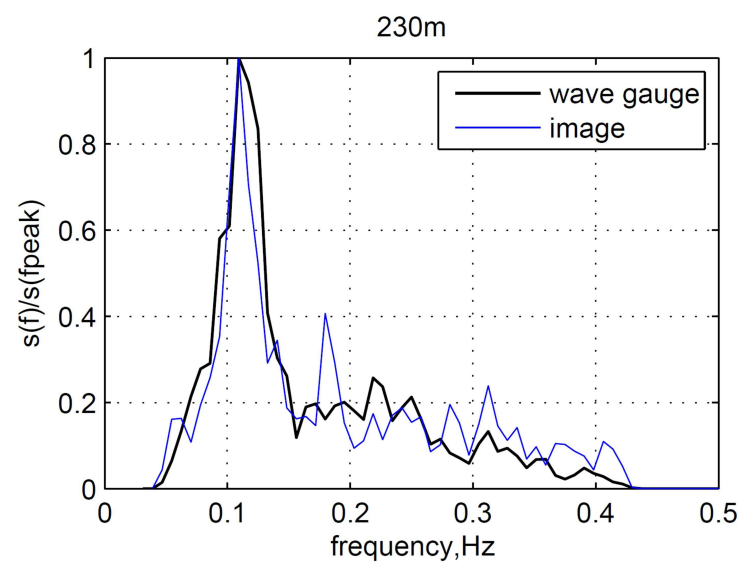

Figure 7. Comparison of wave spectrum from video images and a wave gauge recorded on 18 August 2006 at $09.00 \mathrm{~h}$

Next, we use polygon array design from brightness intensity of pixel data to estimate directional wave spectra at a distance of 230-240 m from shoreline. High-resolution estimation of wave directional spectra require proper layout of pixels in an array at the location of interest to minimize some errors in the cross-power spectra analysis. The importance of the configuration of the array to determine the directional spectra is also reported by Young (1994), among others, who investigated the performance of wave measuring systems. Details on optimizing array layouts design can be seen in Goda (1975).

In this work, the distance between pixels from the optical instruments was set as $D / L=0.2$, where $L$ is the wavelength of the examined component wave. The dimension $D$ was set to typical expected wavelength based on peak frequency and water depth on linear wave theory. For the Hasaki site, $D$ was designed for wave period where $T=8$ seconds in water depth of $2 \mathrm{~m}$. The wavelength was approximately 30-35 meters. With the distance between pixels $(D)$ as $5 \mathrm{~m}$, these waves could be well resolved. The estimated directional wave spectra are shown in Fig. 8. Figure 8 shows the directional wave spectra estimated by the BDM (Bayesian Directional Method) and the EMLM (Extended Maximum Likelihood Method) (Isobe et al. 1984) for comparison.
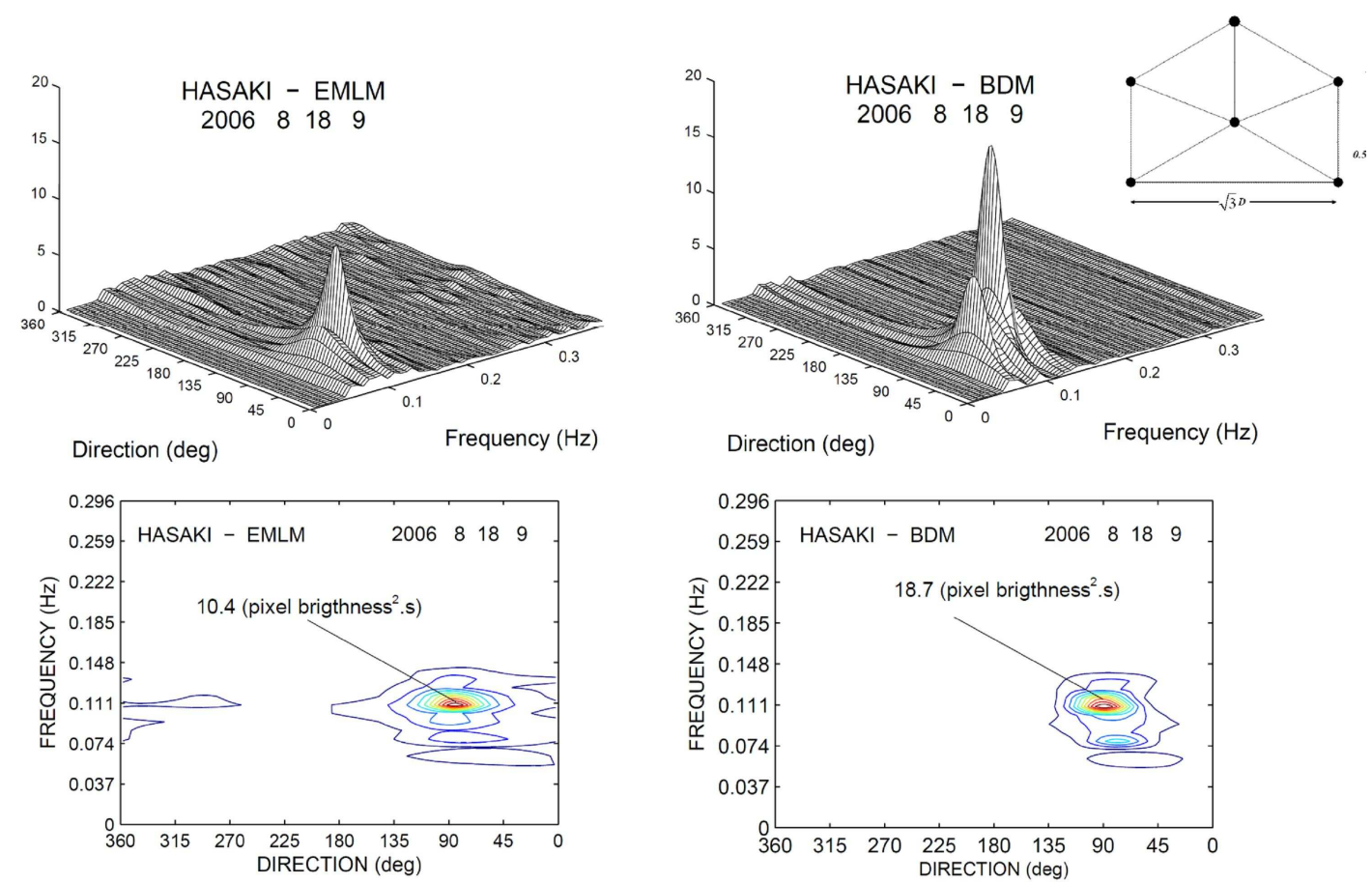

Figure 8. Directional wave spectra estimated from video images in shallow water areas. 
The above figure shows that both directional wave spectra have clear peaks. Directional wave spectra computed by the BDM has a narrow directional function with a higher energy peak compared to the EMLM. Also, energy leakage is present on the directional spreading estimated by the EMLM. It is clearly shown that the accuracy of the estimated directional spectra was improved by using the BDM method. For mean direction estimation, Fig. 8 indicates that both methods estimate similar mean direction about 86 degrees from the shoreline, which indicates the wave approaches perpendicularly to the shoreline.

Due to the lack of near-shore wave measurement data in shallow water waves, in this study, a numerical model of SWAN was selected to verify the results of directional wave spectrum from video images in shallow water. This is because it is important to verify the results of directional wave spectra from video images using whatever data are available or even with different formulations such as using a numerical model.

Figure 9 shows directional wave spectra generated from SWAN model at a cross-shore location 230-240 $\mathrm{m}$ from shoreline. For comparison with directional wave spectra, the results show that there is a good agreement between directional wave spectra in shallow water estimated by the SWAN and video image data. Both methods indicate that the energy distribution of directional spectrum is significantly concentrated in frequency and direction, which is typical of swell waves as indicated in Fig. 9 and Fig. 10 below.
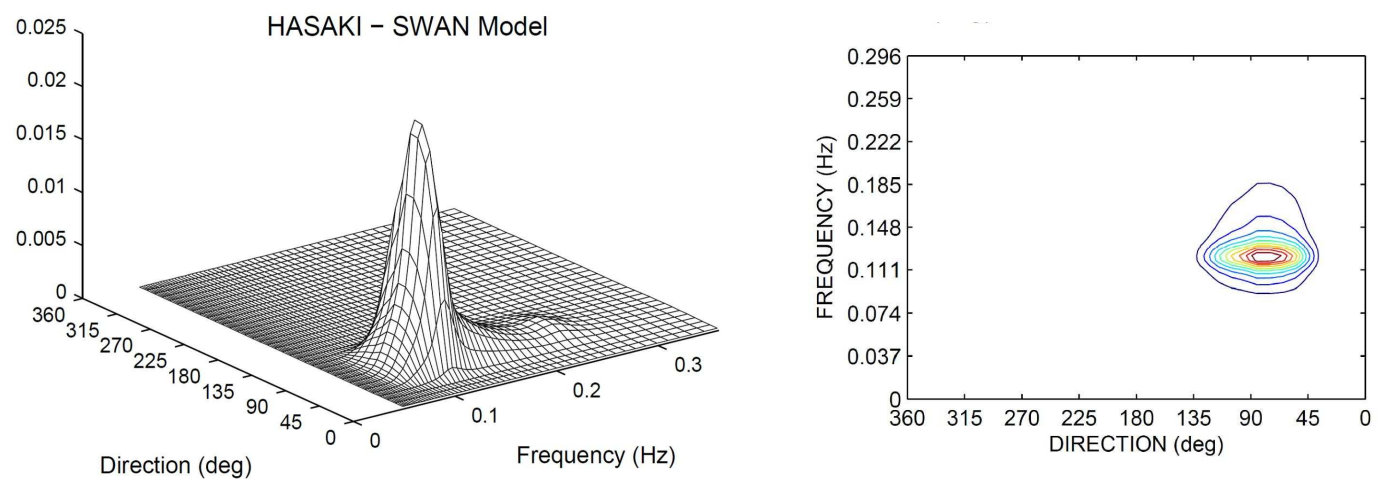

Figure 9. Directional wave spectra calculated by the SWAN model.
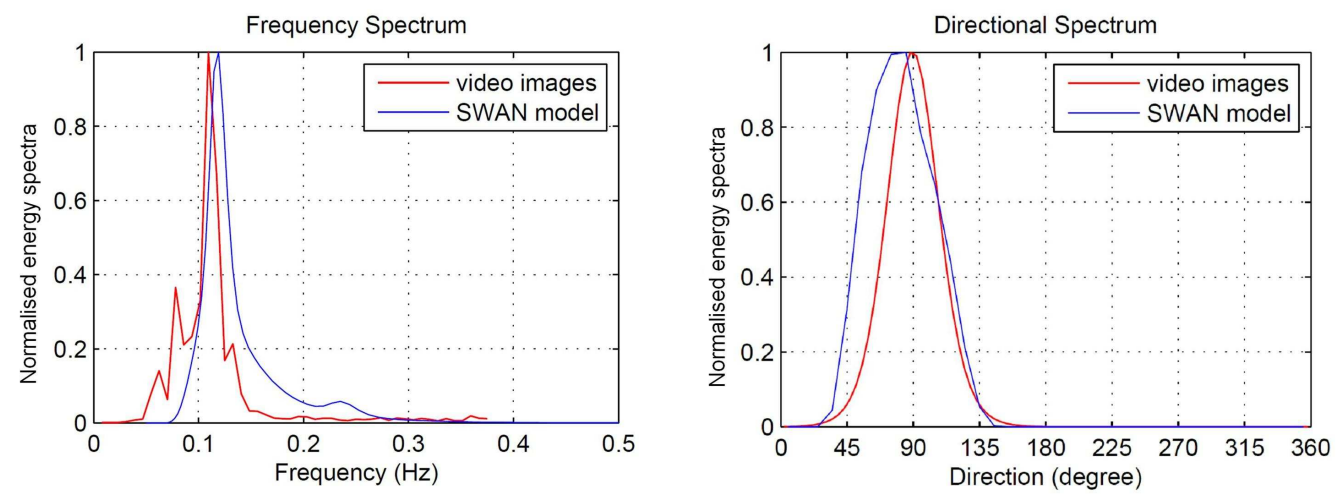

Figure 10. Normalized frequency spectrum and directional spreading estimated using the SWAN model (blue line) and from video images data (red line). 


\section{Non-linear Bathymetry Inversion}

In this section, video images data was used to retrieve shallow water bathymetry based on the nonlinear inversion method for wavenumber estimation. The bathymetry inversion method requires time series data of pixel brightness at an array along a cross-shore and longshore transect in the rectified image sequences as shown in Fig. 10.

Using time series of pixel brightness (timestack) data collected on August 25, 2006, as presented in Fig. 10, the estimated wavenumber was computed at a series of wave frequencies ranging from $0.08 \mathrm{~Hz}$ to $0.11 \mathrm{~Hz}$. It is expected to find the optimum wave component with strong signal from those wave frequency resolutions. On this date, the peak wave period based on field measurement was 9.1 seconds; the wave direction was approach from 81 degrees from North direction; and the significant wave height was $1.01 \mathrm{~m}$.
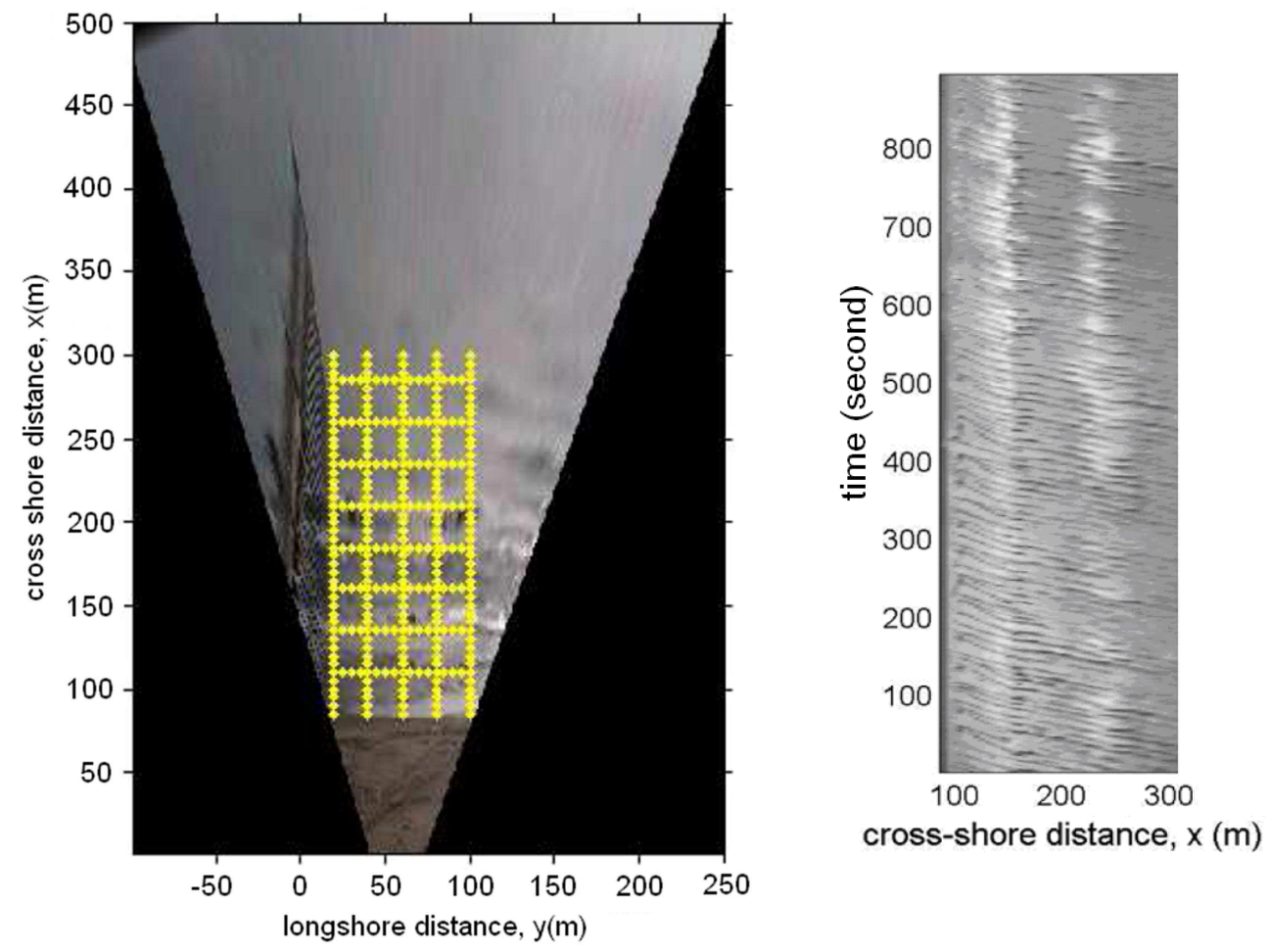

Figure 10. A rectified image with cross-shore and longshore arrays (left). Example of timestack image along cross-shore array at $y=20 \mathrm{~m}$ (right).

The estimated wavenumber was computed for each frequency using the measured bathymetry and the tidal level at the time of the image collection. The non-linear inversion method of LevenbergMarquardt (LM) was applied to the sample cross-spectral correlation at each frequency over the entire array. Figure 11 shows the results of the estimated wavenumber for each sample frequency. As shown in table below, the best of the estimated wavenumbers were obtained at frequency of $0.09 \mathrm{~Hz}$ that showed highest coherence with RMS error of $0.0342 \mathrm{~m}-1$. This frequency $(f=0.09 \mathrm{~Hz})$ corresponds with the peak period of the field measurement.

\begin{tabular}{|c|c|c|c|}
\hline \multicolumn{4}{|c|}{ Table Coherences and RMS errors of wavenumber } \\
\hline No & Frequency & Coherence & RMS wavenumber error \\
\hline 1 & 0.08 & 128.745 & 0.0358 \\
2 & 0.09 & 132.153 & 0.0342 \\
3 & 0.10 & 131.161 & 0.0354 \\
4 & 0.11 & 118.333 & 0.0421 \\
\hline
\end{tabular}



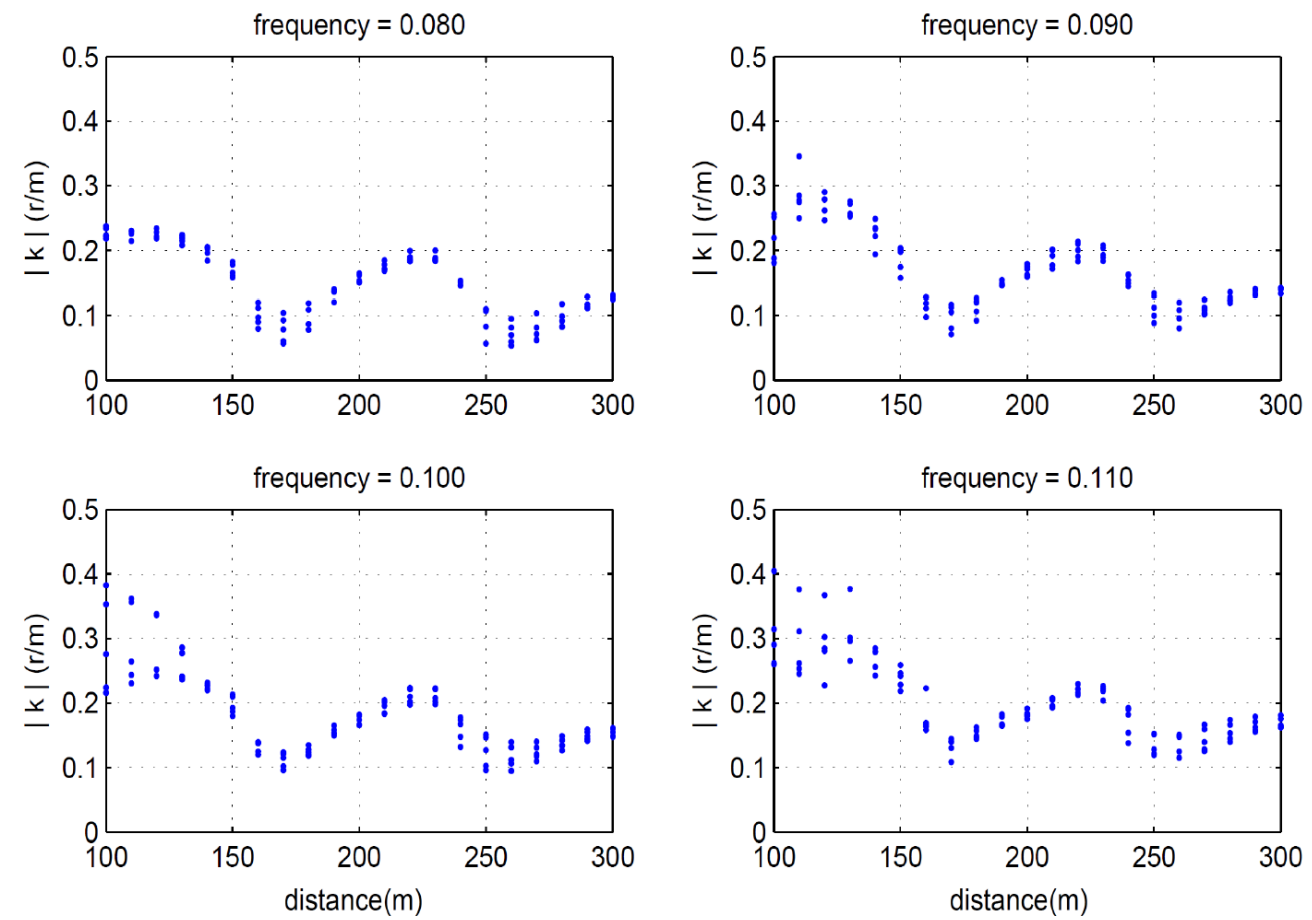

Figure 11. Example of the estimated wavenumber using the non-linear inversion model

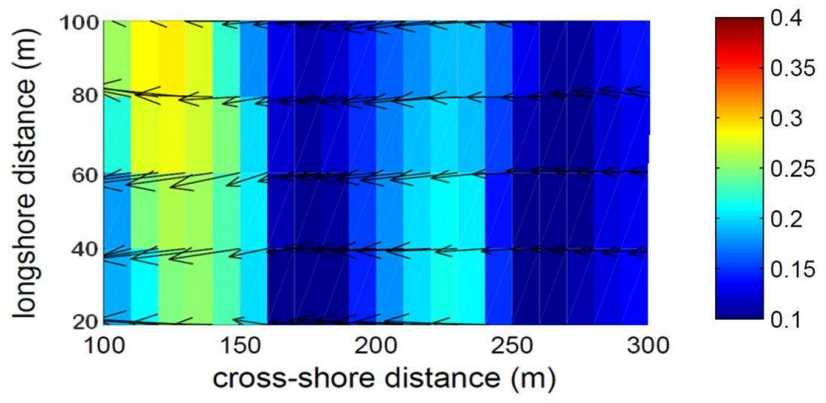

Figure 12. Map of the estimated wavenumber and wave directions (arrows) estimated by the non-linear inversion model at frequency of $0.09 \mathrm{~Hz}$

In addition, the result of bathymetry inversion method based on timestack data by relating wavenumber parameters using the dispersion equation is presented in Fig. 13. Figure 13 shows map of the estimated bathymetry from video images and the bathymetry error prediction from the nonlinear inversion method. The estimated bathymetry by inverted all frequencies shows that the Hasaki Beach has a sand bar at $x=200-250 \mathrm{~m}$. The predicted errors of the estimated bathymetry are accurate with small errors at almost areas as shown in Fig. 13.

The performance of the non-linear bathymetry inversion model is evaluated in comparison with the measured bathymetry. Figure 14 shows comparison of bathymetry profile between the estimated bathymetry profile and the measured bathymetry profile at longshore distance, $y=100 \mathrm{~m}$. It shows that the prediction is the most accurate near shoreline and sand bar, where the differences between estimated and surveyed bathymetry is less than $10-30 \mathrm{~cm}$. 

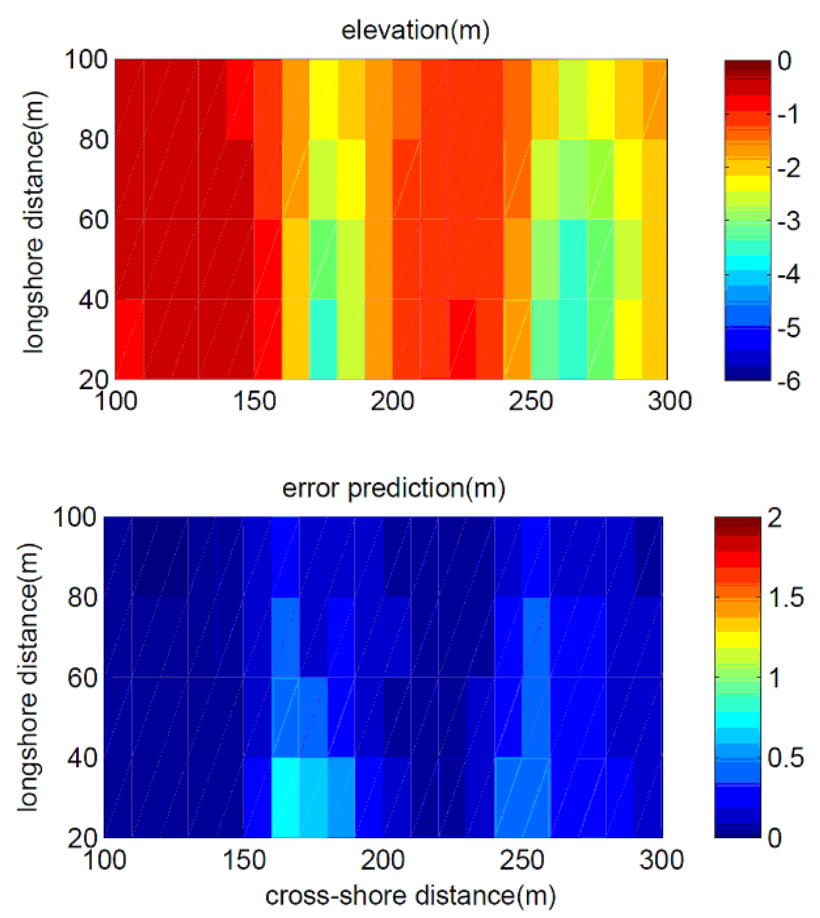

Figure 12. Map of bathymetry derived from video images using the non-linear inversion model (top) and the predicted errors map from the bathymetry inversion (bottom)

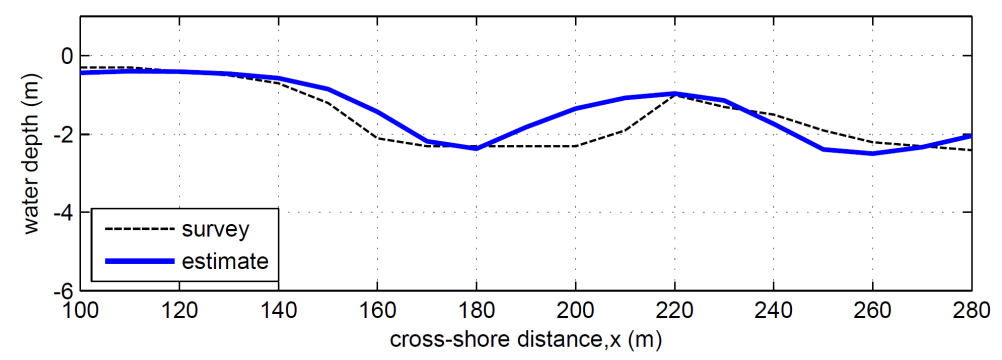

Figure 13. Comparison of bathymetry profiles (at $y=100 \mathrm{~m}$ ) based on video images (solid line) and survey measurements (dot line).

\section{CONCLUSIONS}

We have presented the applicability of video image analysis methods to estimate directional wave spectra and bathymetry in shallow water areas using video images data. These video images data were obtained from measurement made with a single digital camera at Hasaki Beach in Japan. In this study, the BDM is used to estimate directional wave spectra using the group of pixels brightness on video images and non-linear bathymetry inversion is used to estimate bathymetry using time series of pixel brightness intensity at cross-shore and longshore array.

It was found that the BDM can successfully estimate directional wave spectra in shallow water areas and the energy distribution of directional wave spectra in shallow water areas was concentrated in frequency and direction. This directional wave spectrum retrieved from video images was similar to the directional wave spectrum calculated by the SWAN model. In addition, our case study showed that the non-linear inversion model can produce accurate the estimated bathymetry in very shallow water areas. The estimated bathymetry near shoreline and breaking area was estimated reasonable accurate, including the presences of sandbar.

Although the results have some uncertainties due to the limitations on the video image methods, it is shown that video image methods can be very useful and promising technique in order to understand the behavior of the wave field in shallow water areas. 


\section{ACKNOWLEDGMENTS}

The authors wish to thank Port and Airport Research Institute (PARI), Japan for their data support during this study. A special thanks to Nathaniel G. Plant (USGS) for his computer program. Permission to use this code is appreciated. This work was partly supported by Grant-in-Aid for Scientific Research (No. 23360216) from the Japan Society for Promotion of Science (JSPS). Personally, I would also like to acknowledge a scholarship provided by Ditjen Pendidikan Tinggi (DIKTI), Indonesia.

\section{REFERENCES}

Aarninkhof, S.G.J. and Holman, R.A. 1999. Monitoring the nearshore with video, Backscatter, 10(2), 8-11.

Akaike, H. 1980. Likelihood and Bayes procedure, in Bayesian Statistics, University Press, Valencia, 143-166.

Chou, C.R., Yim, J.Z., and Huang, W.P. 2004. Determining the hydrographic parameters of the surface of water from the image sequences of a CCD camera, Experiments in Fluids, 36(4), 515-527.

Dean, R.G., and R.A. Dalrymple. 1991. Water Waves Mechanics for Engineer and Scientist, World Sci., River Edge, N.J., 353 pp

Davidson, M., Koningsveld, M.V., de Kruif, A., Rawson, J., Holman, R., Lamberti, A., Medina, R., Kroon, A. \& Aarninkhof, S. 2007. The CoastView project: developing video-derived Coastal Site Indicators in support of coastal zone management, Coastal Engineering, 54, 463-475.

Goda, Y. 1985. Random Seas and Design of Maritime Structures, University of Tokyo Press, 323 pp.

Hashimoto N., K. Kobune and Y. Kameyama. 1987. Estimation of directional spectrum using the Bayesian approach, and its application to field data analysis, Report of P. H. R. I., 26(5), 57-100.

Holman, R.A. and Stanley, J. 1991. The history and technical capabilities of Argus, Coastal Engineering, 54, 477-491.

Holland, K.T. \& Holman, R.A. 1997. Video estimation of foreshore topography using trinocular stereo, Journal of Coastal Research, 13(1), 81-87.

Holland, K.T., R.A. Holman, T.C. Lipmann, J. Stanley and Plant. 1997. Practical use of video imagery in nearshore oceanography, IEEE J. Oceanic Engineering, 22(1), 81-92.

Holman, R.A. and Chickadel, C. 2004. Optical remote sensing estimates of the incident wave angle field during NCEX, Proceeding of $29^{\text {th }}$ International Conference of Coastal Engineering, ASCE, 1072-1081.

Isobe, M., K. Kondo, and K. Horikawa. 1984. Extension of MLM for estimating directional wave spectrum, in Proc. Symp. On Description and Modeling of Directional Seas, A-6, 57-100.

Lippmann, T.C. and Holman, R.A. 1989. Quantification of sand bar morphology: A video technique based on wave dissipation, Journal of Geophysical Research, 94(C1), 885-1011.

Press, W.H., Teukolsky, S.A., Vetterling, W.T., and Flannery, B.P. 1992. Numerical Recipes in C: The Art of Scientific Computing, 2nd edition, Cambridge University Press.

Plant, N.G. and Holman, R.A. 1997. Intertidal beach profile estimation using video images, Marine Geology, 140, 1-24.

Plant, N.G., K.T. Holland, \& M.C. Haller. 2007. Development of wave number estimation methods applied to coastal motion imagery, IEEE Transactions on Geosciences and Remote Sensing, 25p.

Stockdon, H.F. and Holman, R.A. 2000. Estimation of wave phase speed and nearshore bathymetry from video imagery, Journal of Geophysical Research, 105, 22015-22033.

Young, I.R. 1994. On the measurement of directional wave spectra, Applied Ocean Research, 16, 283294. 